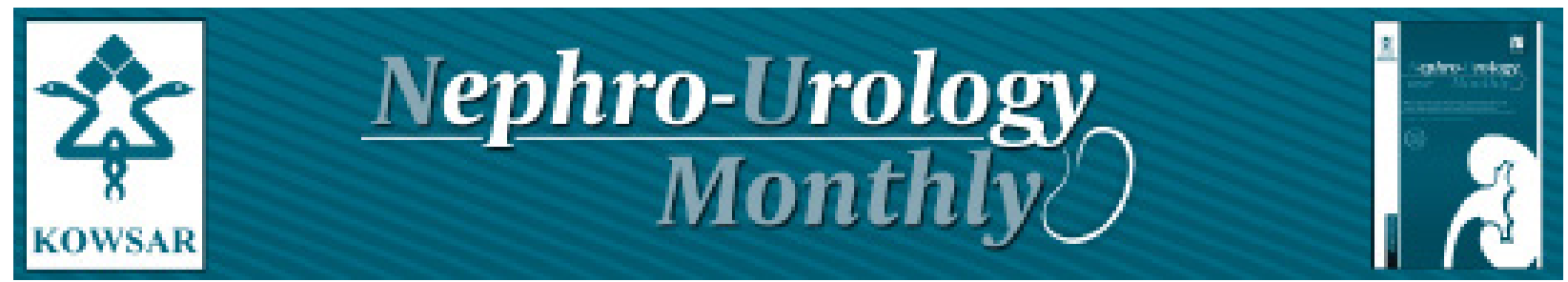

\section{Does Living Donor Hyperoxia Have an Impact on Kidney Graft Function After Transplantation}

\author{
Zohreh Rostami ${ }^{1, *}$, Behzad Einollahi ${ }^{1}$, Mohammad Hassan Ghadiani ${ }^{2}$ \\ ${ }^{1}$ Nephrology and Urology Research Center, Baqiyatallah University of Medical Sciences, Tehran, IR Iran \\ ${ }^{2}$ Shahid Beheshti University of Medical Sciences, Tehran, IR Iran \\ *Corresponding author: Zohreh Rostami, Nephrology and Urology Research Center, Baqiyatallah University of Medical Sciences, Tehran, IR Iran. Tel: +98- \\ 9121544897, Fax: +98-2181262073, E-mail: rostami@numonthly.com.
}

\begin{abstract}
A B S T R A C T
Background: Improvement in the outcome of organ transplantation is related to advances in patient selection criteria, organ preservation, operative techniques, perioperative care and efficacy of immunosuppressive agents.

Objectives: We aimed to evaluate the effects of higher levels of arterial $\mathrm{PaO} 2$ in donors on DGF (delayed graft function).

Patients and Methods: Forty patients over 18 years old with stage 4-5 chronic kidney disease (CKD) who received a kidney from living donors were enrolled. They were randomly grouped in to the case $(n=17)$ and control $(n=23)$ groups and were followed for 2 weeks after transplantation. Donors were exposed to 60\% oxygen for at least 2 hours with a face-mask (venture mask) for 2 consecutive days before transplantation until arterial oxygen pressure increased in arterial blood gas to $200 \mathrm{mmHg}$. Neutrophil Gelatinase Associated Lipocalin (NGAL), Interleuk-18 (IL-18), tumor necrosis factor- $\alpha$ (TNF- $\alpha$ ) and transforming growth factor- $\beta$ (TGF- $\beta$ ) could be good biomarkers for early diagnosis of kidney injury in renal transplant recipients; we assessed kidney function with these biomarkers.

Results: Forty living kidney transplantations including 17 cases and 23 controls were performed; female gender was more prevalent in recipients $(n=16,40 \%)$. The mean age of recipients was $36.1 \pm 12.4(18-67)$ years old. DGF was detected in $2(5.95 \%)$ individuals, from whom one was in the case group and the other one in the control group. In the univariate analysis, there was no significant correlation between age and biomarkers in urine and serum unless for the second serum NGAL $(P=0.02, r=-0.06)$ and second urine IL 18 ( $P=0.03$, $r=-0.5)$ which had a negative correlation, and first urine TNF $\alpha(P=0.02, r=0.7)$ which had a positive correlation.
\end{abstract}

Conclusions: Oxygen therapy in the case group had no significant impact on protection from DGF.

Keywords: Oxygen Inhalation Therapy; Kidney; Transplantation; Delayed Graft Function

Copyright @ 2013, Nephrology and Urology Research Center; Published by Kowsar Corp.

Article type: Research Article; Received: 20 Apr 2013; Accepted: 06 May 2013; Epub: 19 Jun 2013; Ppub: 01 Jul 2013

-Implication for health policy/practice/research/medical education:

We want to evaluate a preconditioning protocol for deceased donation in kidney transplantation.

PPlease cite this paper as:

Rostami Z, Einollahi B, Ghadiani MH. Does Living Donor Hyperoxia Have an Impact on Kidney Graft Function After Transplantation. Nephro Urol Mon.2013;5(3): 835-9. DOI: 10.5812/numonthly.11870

Copyright ( ) 2013, Nephrology and Urology Research Center; Published by Kowsar Corp.

This is an Open Access article distributed under the terms of the Creative Commons Attribution License (http://creativecommons.org/licenses/by/3.0), which permits unrestricted use, distribution, and reproduction in any medium, provided the original work is properly cited. 


\section{Background}

Parallel to the rapid growth of the incidence of end stage renal disease (ESRD) (1), demand for kidney transplants has grown faster than the actual supply of kidneys (2). However, ESRD has been estimated to be between 15 and 80 per one million individuals (3). Deceased donor kidney transplants offer an additional possibility for patients requiring renal replacement therapy. Nevertheless, the improvement in the outcome of organ transplantation is related to advances in patient selection criteria, organ preservation, operative techniques, perioperative care and efficacy of immunosuppressive agents (4).

Delayed graft function (DGF) defined by the need for dialysis within the first week post-transplantation, portends a foreshortened allograft survival of $73 \%$ by the first year compared to $83 \%$ for recipients who do not require dialysis (5). The reported incidence of DGF from various centers ranges from 13.3 to 52 \% (5). Organ preservation has an impact on allograft renal function (2). On the other hand, hyperbaric oxygen (HBO) therapy has been revealed to modulate both cellular and humoral immune response as well as lessen the severity of reperfusion injury (2). However, there is no evidence for the harmful effect of oxygen challenge via invasive mechanical ventilation on the risk of DGF in deceased donors before recovery of kidney.

\section{Objectives}

Although, oxygen is a vital substrate in the reduction of hypoxia, anoxia and ischemia, it also acts as a toxic metabolite due to reactive oxygen species (ROS) production $(2,3)$. On the other hand, elevated oxygen tension during reperfusion may actually promote necrosis (6).

Regarding this controversy, the authors attempted to evaluate the effects of higher levels of arterial $\mathrm{PaO} 2$ in donors on DGF. Also, this paper provides an overview of the relationship between oxygen therapy and DGF and the potential harmful impacts of oxygen therapy in kidney transplantation.

\section{Patients and Methods}

\subsection{Participants}

This cross-sectional study was initiated after the protocol was approval by the Baqiyatallah Medical University Ethics Committee and informed consent was obtained regularly from all recipients and donors between 2006 and 2007.

Forty patients over 18 years of age with stage 4-5 chronic kidney disease (CKD) who received a kidney from a living donor were enrolled. They were randomly grouped in to the case $(n=17)$ and control $(n=23)$ groups and were followed for 2 weeks after transplantation.

The recipients who had long ischemic time, vascular problems, hemodynamic instability during operation, history of smoking and diabetes were excluded. In addition, donors with past medical history of pnumothrax, asthem, seizures, optic neuritis, middle ear surgery and heavy smokers were excluded.

The immunosuppressive protocol was a triple therapy initiated by cyclosporine ( $6 \mathrm{mg} / \mathrm{kg} /$ day $)$, mycophenolate mofetil (1 g/day) and prednisolone ( $2 \mathrm{mg} / \mathrm{kg} /$ day). As neutrophil gelatinase associated lipocalin $(\operatorname{NGAL})(7,8)$, interleuk-18 (IL-18), tumor necrosis factor- $\alpha$ (TNF- $\alpha$ ), transforming growth factor- $\beta$ (TGF- $\beta$ ) could be good biomarkers for early diagnosis of kidney injury in renal transplant recipients, we assessed kidney function with these biomarkers.

\subsection{Oxygen Protocol}

Donors were exposed to 60\% oxygen for at least 2 hours with a face-mask (venture mask) for 2 consecutive days before transplantation until arterial oxygen pressure increased in arterial blood gas to $200 \mathrm{mmHg}$.

\subsection{Variables}

Following variables were gathered for all recipients: age, gender and urine NGAL, IL-18, TNF- $\alpha$, TGF- $\beta$ for consecutive days.

\subsection{Definitions}

DGF was defined as dialysis requirement in the first week after kidney transplantation (9).

\subsection{Statistical Analysis}

Data were analyzed with SPSS, version 17.0. Quantitative variables have been expressed as mean \pm SD and qualitative variables have been shown by percentages. Continuous data were compared by Student's t-test and categorical data were analyzed using the Chi-square or Fisher's exact test. P values less than 0.05 were significant.

\section{Results}

\subsection{General Characteristics}

Forty living kidney transplantations including 17 cases and 23 controls were performed; female gender was more prevalent in recipients ( $n=16,40 \%)$. In the case group male: female ratio was 1:2.4 while in the control group, this was 1.3:1 $(\mathrm{P}=0.1)$. The mean age of recipients was 36.1 \pm 12.4 (18-67) years old. DGF was detected in $2(5.95 \%)$ individuals, of whom one was in the case group and the other one in control group. Mean of serum and urine detected biomarkers are summarized in Table 1. Nonparametric correlation between age and urine or blood kidney injury biomarkers after transplantation in the case group is summarized in Table 2. 
Table 1. Mean and Standard Deviation of Serum and Urine

Kidney Injury Biomarkers During 4 Days

\begin{tabular}{|c|c|}
\hline Biomarker & Mean $\pm S D(\min -\max )$ \\
\hline Urine NGAL $1^{\mathrm{a}}$ & $534.55 \pm 460.8(5-1190)$ \\
\hline Urine NGAL 2 & $227.27 \pm 366.001(5-1215)$ \\
\hline Urine NGAL 3 & $100.38 \pm 187.4(5-710)$ \\
\hline Urine NGAL 4 & $124.17 \pm 152.52(25-430)$ \\
\hline Serum NGAL 1 & $788.95 \pm 535.19(40-1900)$ \\
\hline Serum NGAL 2 & $395.38 \pm 438.35(910-1940)$ \\
\hline Serum NGAL 3 & $325.2 \pm 230.9(915-1010)$ \\
\hline Serum NGAL 4 & $237.89 \pm 225.55(10-980)$ \\
\hline IL 18, $\mathbf{1}^{\mathrm{a}}$ & $10.19 \pm 1714.6(0-5000)$ \\
\hline IL 18, 2 & $1148.2 \pm 1711.2(0-5000)$ \\
\hline IL 18, 3 & $1211.38 \pm 1582.6(115-5000)$ \\
\hline IL 18, 4 & $1177.73 \pm 1496.6(0-5000)$ \\
\hline TNF $\alpha, \mathbf{1}^{\mathrm{a}}$ & $31.75 \pm 37.8(0-120)$ \\
\hline TNF $\alpha, 2$ & $96 \pm 177.2(10-680)$ \\
\hline TNF $\alpha, 3$ & $208.9 \pm 313.74(10-1000)$ \\
\hline TNF $\alpha, 4$ & $279.6 \pm 313.9(12-1000)$ \\
\hline TGF $\beta, \mathbf{1}^{\mathrm{a}}$ & $4760 \pm 3669.76(0-12000)$ \\
\hline TGF $\beta, 2$ & $4104.17 \pm 2703.18(470-9000)$ \\
\hline TGF $\beta, 3$ & $2705.38 \pm 2208.15(0-8000)$ \\
\hline TGF $\boldsymbol{\beta}, \mathbf{4}$ & $3900.6 \pm 2480.15(220-8000)$ \\
\hline
\end{tabular}

\subsection{Univaritate Analysis}

In the univariate analysis, there was no significant correlation between age and biomarkers in urine and serum unless for the second serum NGAL $(\mathrm{P}=0.02, \mathrm{r}$ $=-0.06)$, second urine IL $18(P=0.03, r=-0.5)$ which had a negative correlation, and first urine TNF- $\alpha(\mathrm{P}=$ $0.02, r=0.7)$ which had a positive correlation. Oxygen therapy in the case group had no significant impact on protection from DGF.

\section{Discussion}

In the current study, we studied the effect of donor hyperoxia ( $\mathrm{PaO} 2 \geq 200 \mathrm{mmHg}$ ) on kidney function after kidney transplantation. Despite the mentioned beneficial effects of hyperbaric oxygen (HBO), we revealed that donor hyperoxia, was not associated with advantages. Einollahi et al. in 2011 (4) showed that this level of hyperoxia resulted in 5 folds probability for DGF presentation compared to $\mathrm{PaO} 2$ less than 200 $\mathrm{mmHg}$ in deceased donor. According to the previous studies $(3,10,11)$, hyperbaric oxygen exposure can preserve organs in a more optimal condition for trans- plantation. Although experimental studies have suggested that hyperoxia induced by hyperbaric oxygen may be favorable in the management of reperfusion injury, its mechanism remains unclear (10). However, there are also reports on the immunomodulatory effects of hyperbaric oxygen, which have been directed towards its effect on ischemia reperfusion injury (IRI) $(12,13)$. Although researchers have also demonstrated a suppressive effect on both humoral and cell mediated immunity in animal studies (3) as well as alternation in cell surface MHC class I antigen expression $(3,14)$, yet HBO has been noted to increase ROS production $(15,16)$.

Moreover, the deteriorative outcome of pre-operation hyperoxia on DGF may be described by the toxic impact of oxygen-free radicals released through reperfusion of the ischemic region (17). Oxygen-free radicals participate in lipid peroxidation and lead to endothelium and cell membrane injury, ensuing in a total defect of cellular integrity. On the other hand, cell cannot be metabolized aerobically anymore which leads to a progressive energy reduction and cytosolic $\mathrm{Ca}^{++}$overload. Reperfusion of the graft in the presence of decreased cellular energy may prone further damage resulting in cell death and possible renal allograft failure (10). In addition, it seems that reperfusion not only leads to energy deficiency within the kidney and endothelial cells as well as the products of reactive oxygen species (ROS) but also promotes release of potent inflammatory cytokines such as tumor necrosis factor- $\alpha$ (TNF- $\alpha$ ) and interleukin- 1 (IL-1) (18) as well as factors such as the pro-apoptotic tumor protein p53 gene (TP53) and the anti-apoptotic, antioxidant heme oxygenase 1 gene (HMOX1) that play an important role in modulating apoptosis. Though in acute kidney injury the TNF- $\alpha$ gene, transforming growth factor $\beta 1$ gene (TGF- $\beta 1$ ), and interleukin 10 gene (IL-10) have more important effects on regulation of inflammatory response (5). In kidney allograft, cell adhesion molecules are expressed by TNF- $\alpha$ and promote kidney damage (19-21). On the other hand, there is greater expression of TNF- $\alpha$ in kidney allografts with DGF than those without DGF (5). Therefore, donor hyperoxia that enhances TNF- $\alpha$ activity could predispose recipients to DGF (11). On the basis of previous studies, although HBO preserves tissue oxygenation but immunomodulatory effect of HBO therapy has been attributed predominantly to the hyperbaric effect rather than to hyperoxia $(3,22)$. Animal studies have substantiated an inhibitory effect of HBO therapy in the absence of hyperoxia on IFN- $\gamma$. Subsequently, despite of the increase in oxygen tension, hyperbaric oxygen affects cellular cytoskeleton, leading to a reduction in IFN- $\gamma$. In other words mechanism of HBO immunomodulation is different from energy formation (3). 
Table 2. Nonparametric Correlation Between Age and Urine or Blood Kidney Injury Biomarkers After Transplantation in Case Group During 4 Days

\begin{tabular}{lll}
\hline & Correlation $(\mathbf{r})$ & $\mathbf{P}$ \\
\hline Urine NGAL 1 $^{\text {a }}$ & 0.08 & 0.8 \\
\hline Urine NGAL 2 & 0 & 1 \\
\hline Urine NGAL 3 & 0.4 & 0.7 \\
\hline Urine NGAL 4 & - & - \\
\hline Serum NGAL 1 & 0.3 & 0.4 \\
\hline Serum NGAL 2 & -0.6 & 0.02 \\
\hline Serum NGAL 3 & -0.03 & 0.9 \\
\hline Serum NGAL 4 & -0.6 & 0.05 \\
\hline IL 18, $\mathbf{1}^{\text {a }}$ & -0.6 & 0.07 \\
\hline IL 18, 2 & -0.5 & 0.03 \\
\hline IL 18, 3 & -0.2 & 0.5 \\
\hline IL 18, 4 & 0.1 & 0.6 \\
\hline TNF $\boldsymbol{\alpha}, \mathbf{1}^{\text {a }}$ & 0.7 & 0.02 \\
\hline TNF $\boldsymbol{\alpha}, \mathbf{2}$ & 0.6 & 0.07 \\
\hline TNF $\boldsymbol{\alpha}, \mathbf{3}$ & 0.5 & 0.6 \\
\hline TNF $\boldsymbol{\alpha}, \mathbf{4}$ & 0.03 & 0.9 \\
\hline TGF $\boldsymbol{\beta}, \mathbf{1}^{\text {a }}$ & 0.07 & 0.8 \\
\hline TGF $\boldsymbol{\beta}, \mathbf{2}$ & -0.7 & 0.1 \\
\hline TGF $\boldsymbol{\beta}, \mathbf{3}$ & -0.7 & 0.1 \\
\hline TGF $\boldsymbol{\beta}, \mathbf{4}$ & 0.2 & 0.4 \\
\hline a Abbreviations: NGAL, neutrophil gelatinase associated lipocalin; IL- \\
\hline 18, Interleuk-18; TNF- $\alpha$, tumor necrosis factor- & $\alpha ;$ TGF- $\beta$, transforming \\
\hline growth factor- $\beta$ & & \\
\hline
\end{tabular}

The other mechanism of this toxic effect of HBO might be associated with lipid peroxidation which, cause cell membrane and organelle damage in the IRI state and level of injury augments during exposure to normobaric $100 \%$ oxygen $(3,15,16)$. Therefore, it seems that oxygen saturation is more important than oxygen pressure in organ damage and DGF induction.

Limitations: it is possible that the association of kidney injury with other predictors were not seen in our study. The little sample size is the most important limitation in our study.

Conclusion: The current study revealed that normobaric hyperoxia of living donors before kidney transplantation has no effect on kidney function in renal transplant recipients. Further studies are required to confirm our findings.

\section{Acknowledgements}

The authors would like to thank Baqiyatallah hospital kidney transplantation ward.

\section{Authors' Contribution}

Dr Einollahi: design and data analysis, Dr Rostami: writ- ing, Dr Ghadiani: data collection.

\section{Financial Disclosure}

The authors declare that there are no conflicts of interest.

\section{Funding/Support}

Nephrology and Urology Research Center, Baqiyatallah University of Medical Sciences, Tehran, Iran.

\section{References}

1. Gilbertson DT, Liu J, Xue JL, Louis TA, Solid CA, Ebben JP, et al Projecting the number of patients with end-stage renal disease in the United States to the year 2015. J Am Soc Nephrol. 2005;16(12):3736-41.

2. Muralidharan V, Christophi C. Hyperbaric oxygen therapy and liver transplantation. HPB (Oxford). 2007;9(3):174-82.

3. Israni AK, Li N, Cizman BB, Snyder J, Abrams J, Joffe M, et al. Association of donor inflammation- and apoptosis-related genotype and delayed allograft function after kidney transplantation. Am Kidney Dis. 2008;52(2):331-9.

4. Einollahi B, Poor-Reza-Gholi F, Rezaeean S, Jahani Y, Nafar M, Najafizadeh K, et al. Deceased-donor hyperoxia deteriorates kidney graft function.J Europ Soci Organ Transplant. 2011;24(2):e16-8.

5. Malago M, Hertl M, Testa G, Rogiers X, Broelsch CE. Split-liver transplantation: future use of scarce donor organs. World J Surg. 2002;26(2):275-82.

6. Sterling DL, Thornton JD, Swafford A, Gottlieb SF, Bishop SP, Stanley AW, et al. Hyperbaric oxygen limits infarct size in ischemic rabbit myocardium in vivo. Circulation. 1993;88(4 Pt 1):1931-6.

7. Rostami Z, Nikpoor M, Einollahi B. Urinary Neutrophil Gelatinase Associated Lipocalin (NGAL) for Early Diagnosis of Acute Kidney Injury in Renal Transplant Recipients. Nephro-Urology Monthly. 2013;5(2):745-52.

8. Rostami Z, Heidari F. How neutrophil gelatinase-associated lipocalin can be presented in plasma and urine. Iran J Kidney Dis 2012;6(5):325-7.

9. Parikh CR, Jani A, Mishra J, Ma Q, Kelly C, Barasch J, et al. Urine NGAL and IL-18 are predictive biomarkers for delayed graft function following kidney transplantation. Am J Transplant. 2006;6(7):1639-45.

10. Bayrakci B. Preservation of organs from brain dead donors with hyperbaric oxygen. Pediatr Transplant. 2008;12(5):506-9.

11. Corradini SG, Elisei W, De Marco R, Siciliano M, Iappelli M, Pug liese F, et al. Preharvest donor hyperoxia predicts good early graft function and longer graft survival after liver transplantation. Liver Transpl. 2005;11(2):140-51.

12. Hansbrough JF, Eiseman B. Immunosuppression with hyperbaric oxygen. Surg Forum. 1979;30:313-5.

13. Hansbrough JF, Piacentine JG, Eiseman B. Immunosuppression by hyperbaric oxygen. Surgery. 1980;87(6):662-7.

14. MacKenzie DA, Sollinger HW, Hullett DA. Decreased immunogenicity of human fetal pancreas allografts following hyperbaric oxygen culture. Transplant Proc. 2003;35(4):1499-502.

15. Ozden TA, Uzun H, Bohloli M, Toklu AS, Paksoy M, Simsek G, et al. The effects of hyperbaric oxygen treatment on oxidant and antioxidants levels during liver regeneration in rats. Tohoku J Exp Med. 2004;203(4):253-65.

16. Kurir TT, Markotic A, Katalinic V, Bozanic D, Cikes V, Zemunik T, et al. Effect of hyperbaric oxygenation on the regeneration of the liver after partial hepatectomy in rats. Braz J Med Biol Res. 2004;37(8):1231-7.

17. Novitzky D. Donor management: state of the art. Transplant Proc 1997;29(8):3773-5.

18. Colletti LM, Kunkel SL, Walz A, Burdick MD, Kunkel RG, Wilke $\mathrm{CA}$, et al. The role of cytokine networks in the local liver injury following hepatic ischemia/reperfusion in the rat. Hepatology. 1996;23(3):506-14. 
19. Toback FG. Regeneration after acute tubular necrosis. Kidney Int. 1992;41(1):226-46.

20. Donnahoo KK, Meng X, Ayala A, Cain MP, Harken AH, Meldrum DR. Early kidney TNF-alpha expression mediates neutrophil infiltration and injury after renal ischemia-reperfusion. Am J Physiol. 1999;277(3 Pt 2):R922-9.

21. Daemen MA, van de Ven MW, Heineman E, Buurman WA. Involve- ment of endogenous interleukin-10 and tumor necrosis factoralpha in renal ischemia-reperfusion injury. Transplantation. 1999;67(6):792-800.

22. Granowitz EV, Skulsky EJ, Benson RM, Wright J, Garb JL, Cohen ER, et al. Exposure to increased pressure or hyperbaric oxygen suppresses interferon-gamma secretion in whole blood cultures of healthy humans. Undersea Hyperb Med. 2002;29(3):216-25. 